\title{
Zhihu Live is a New and Effective Public Health Education Method for Pulmonary Tuberculosis Via an Online Community Q\&A Platform
}

\author{
Siyuan Gan ${ }^{1, \# \text {, Wang Liu }}{ }^{2, \#, ~ C a i l i n g ~ H u a n g ~}{ }^{1, \#, ~ S i q i n ~ C h e n ~}{ }^{1}$, Yanqiu Liao ${ }^{1}$, Rujia Li ${ }^{1}$, Yu Zeng ${ }^{2}$, \\ Yanqin Sun', Yanping $\mathrm{Ha}^{1, *}$ \\ ${ }^{1}$ Department of Pathology, Guangdong Medical University, Zhanjiang 524023, China \\ ${ }^{2}$ Department of Respiratory, The Second Affiliated Hospital of Guangdong Medical University, Zhanjiang 524023, China \\ ${ }^{\#}$ These authors contribute equally to this work. \\ *Corresponding Author
}

\begin{abstract}
Background: We hope to develop a new and effective health education method for pulmonary tuberculosis (PTB). Method: We initiated a lecture in an online knowledge-sharing lecture series, "Zhihu Live", on the Zhihu website, which is an online community Q\&A platform. The Zhihu Live lecture examined in the current study was titled "The pathogenesis, treatment and prevention of pulmonary tuberculosis". Results: From April 2018 to June 2021, 1706 people participated in this study. The participants reported that they highly appreciated this Live lecture. Conclusion: Zhihu Live can facilitate the effective learning and spread of knowledge about PTB for TB patients and the people who surround them. Zhihu Live should be considered a useful new method of health education for the public. In addition to TB, the prevention and treatment of other infectious diseases, including HIV/AIDS and COVID-19, could greatly benefit from this innovative health education method.
\end{abstract}

Keywords: Health education, Infectious diseases, Online community, Pulmonary tuberculosis, Zhihu Live.

\section{Introduction}

Pulmonary tuberculosis (PTB) is a global disease that threatens public health worldwide. In 2019, ten million people were diagnosed with PTB and 1.4 million died from the disease worldwide[1]. There is no doubt that ignorance regarding the origin, spread, pathological process, treatment, and prevention of PTB will lead to a high prevalence of the disease[2]. Health education involving PTB is a critical and useful method for the prevention and treatment of tuberculosis (TB)[2],[3]. A previous study found that the treatment adherence of TB patients not only affects the outcome of their therapy and their risk of acquired drug resistance but is also critical for TB treatment[3],[4]. In addition, health education targeted to TB patients promotes their awareness regarding their treatment and thus their treatment adherence[3],[5]. Furthermore, in addition to TB patients, health education targeted to those who surround them, including their patient caregivers, family members, classmates, and colleagues, has been identified as being very important for the treatment and prevention of $\mathrm{TB}[3]$. Social media has been found to be a useful educational tool for promoting public health[6]. With mass media interventions, health education can produce significant positive health changes by enforcing positive health behaviors and avoiding negative health behaviors among individuals[6]. Mass media can transmit health messages through a variety of forms, including digital media[6].

The social media question and answer website Quora has been found to be a good tool for health education involving multiple health issues[7-9]. Zhihu is China's version of Quora. In classical Chinese, "Zhihu" means "Do you know?"[10] As of 2018, the platform had 200 million registered users and over 120 million answers[11]. Zhihu is known as China's most popular question-and-answer platform and the largest knowledge-sharing community[11]. In addition to the Zhihu website, Zhihu users can sign up for the Zhihu app. With the addition of the app, this platform is convenient to use, regardless of whether one is using a personal computer or a cell phone. On this platform, the users create, answer and edit questions. In addition to this basic feature, there are a variety of other features regarding knowledge sharing and dissemination, including Zhihu Live. Zhihu Live is an interactive live streaming session that emphasizes "real-time knowledge sharing and questions and answers". Speakers disseminate the knowledge, opinions or experience within their areas of expertise[11]. Some Live lectures are free, while most of them require a small admission fee to join[10,11]. For a standard Live presentation, the lectures are broadcast live (sound only) and synchronized with associated pictures and text content. The participants can ask questions via written messages and obtain answers from the speaker immediately. The lecturers are required to prepare their presentations carefully, with lectures, pictures, videos and PowerPoint slides. After the Live lecture is finished, the participants can still replay the Live recording. In addition, although the Live lecture has been concluded, new users can still choose to participate in the Live and obtain its content by replaying the Live recording. The performance of lecturers in their Live lectures can be evaluated by participants who rate their performance on a scale that ranges from 1 to 10 . Furthermore, the participants can write comments to evaluate each Live lecture. The score and comments of the Live lectures can be references for new users to consult if they decide to pay for Live access.

We aimed to initiate a new Live lecture titled "The pathogenesis, treatment and prevention of pulmonary tuberculosis" on Zhihu to facilitate the effective learning and spread of knowledge about PTB. This approach should be a useful new method for public health education, especially for TB patients and the people who surround them. 


\section{Method}

We announced the launch 3 weeks prior to the initiation of the Live lecture and included segments such as "Introduction to Live lectures" and "Syllabus of the Live" on the platform. The "Introduction to Live lectures" and "Syllabus of the Live" segments have been shown to attract potential participants who might be interested in the Live lecture. Due to this announcement, Zhihu users would have a chance to notice this Live lecture and consider joining it. In addition to the announcement of the Live lecture, we made a PowerPoint presentation with 79 slides demonstrating the pathogenesis, treatment and prevention of pulmonary tuberculosis. The study was an online lecture for the scientific popularization of pulmonary tuberculosis and does not provide specific diagnosis or treatment plans. Furthermore, the participants can anonymously participate in the Live lecture and ask questions anonymously. The Institutional Ethical Review Board of the affiliated hospital of Guangdong Medical University confirms that ethics approval was not required.

The Live lecture began on April 22, 2018, at 7:30 PM and lasted for 108 minutes. The author was the lecturer. Accompanied by the PowerPoint slides, he first presented the topic "The pathogenesis, treatment and prevention of pulmonary tuberculosis". Then, he answered 8 questions related to the topic.

\subsection{Introduction to Live Lectures}

Pulmonary tuberculosis is the second most common infectious disease in China, regardless of incidence and death. Approximately 550 million people in China have been infected with Mycobacterium tuberculosis. Approximately 1 million new tuberculosis patients are diagnosed each year, and there are approximately 5 million patients with active tuberculosis in the country. Among them, more than $1 / 4$ of the patients are infectious. The spread of tuberculosis is not only greatly harmful to patients but also causes suffering for the patients' relatives, friends and social network.

With accessible language and pictures, this Live lecture will show the causes and spread pathways of pulmonary tuberculosis, the pathogenesis of pulmonary tuberculosis and why it is so difficult to treat. It will also address the question whether one has pulmonary tuberculosis if one is infected with Mycobacterium tuberculosis. Typical symptoms of pulmonary tuberculosis, the principles and precautions for medical treatment, the importance and precautions of adhering to the treatment principles of pulmonary tuberculosis, the causes and related harms of drug resistance in pulmonary tuberculosis, and the issue of how we should scientifically prevent pulmonary tuberculosis will also be addressed.

\subsection{Acknowledgments}

(1) This Live lecture is for the scientific popularization of pulmonary tuberculosis and does not provide specific diagnosis or treatment plans.

(2) Since the content of this Live lecture involves infectious diseases, the participants can anonymously participate in the Live lecture and ask questions anonymously.

\subsection{Syllabus of the Live Lecture}

(1) The cause and mode of transmission of pulmonary tuberculosis.

(2) The pathogenesis of pulmonary tuberculosis. Will one necessarily develop pulmonary tuberculosis if one becomes infected with Mycobacterium tuberculosis?

(3) The treatment difficulty of pulmonary tuberculosis; i.e., why is it so difficult to treat?

(4) Typical symptoms of pulmonary tuberculosis.

(5) Instructions for the medical treatment of tuberculosis, including the selection of treatment institutions, preferential treatment policies, medical reimbursement, the difference between initial treatment and retreatment of pulmonary tuberculosis, etc.

(6) The importance of and precautions related to the treatment principles of pulmonary tuberculosis, the adjustment of one's life and diet during treatment, etc.

(7) Recognizing the hazards of drug resistance and how to avoid drug resistance.

(8) The prevention of pulmonary tuberculosis. How can the general population prevent pulmonary tuberculosis? How can tuberculosis patients prevent transmission? How can one prevent the recurrence of tuberculosis?

(9) Free time for questions.

\subsection{Detailed Flow Chart of the Zhihu Live Lecture}

Characteristics and transmission of Mycobacterium tuberculosis.

The source of tuberculosis infection, and the population susceptible to tuberculosis.

Mycobacterium tuberculosis detection, including the definition and the characteristics and significance of sputum smear examinations and sputum culture examinations.

Definition and significance of smear-negative tuberculosis and smear-negative tuberculosis.

Pathogenic mechanism of Mycobacterium tuberculosis after infection and the characteristics of the pathological changes related to tuberculosis.

Introduction of the reasons for the poor treatment of tuberculosis, including low immunity, insensitivity to treatment drugs, drug resistance and ease of relapse.

Statutory institutions for tuberculosis treatment in China.

Preferential policies for tuberculosis treatment in China.

Definition of the initial treatment of tuberculosis, and the definition of the retreatment of tuberculosis. 
Illustration of the preferential policies for tuberculosis treatment in China.

Detailed introduction of the treatment principles of tuberculosis, including early, combined, appropriate, regular, and full-course treatment, and the importance of such treatments.

Food- and life-related precautions for TB patients.

Definition of, causes of and harms related to resistance.

How should the public prevent tuberculosis? What precautions should people take when they are around tuberculosis patients? How can tuberculosis patients prevent relapse after recovery?

\section{Results}

According to the statistics provided by the Zhihu website, from April 2018 to June 2021, one thousand seven hundred and six people participated in this Live lecture. After joining the Live lecture, twenty-nine participants rated it. The average of rating was 8 (Figure 1, in Chinese). Meanwhile, of those evaluators, twenty-six participants wrote comments about the Live lecture. Most reviewers showed great satisfaction with the content of the Live lecture and the talker's performance (Figure 2, in Chinese).

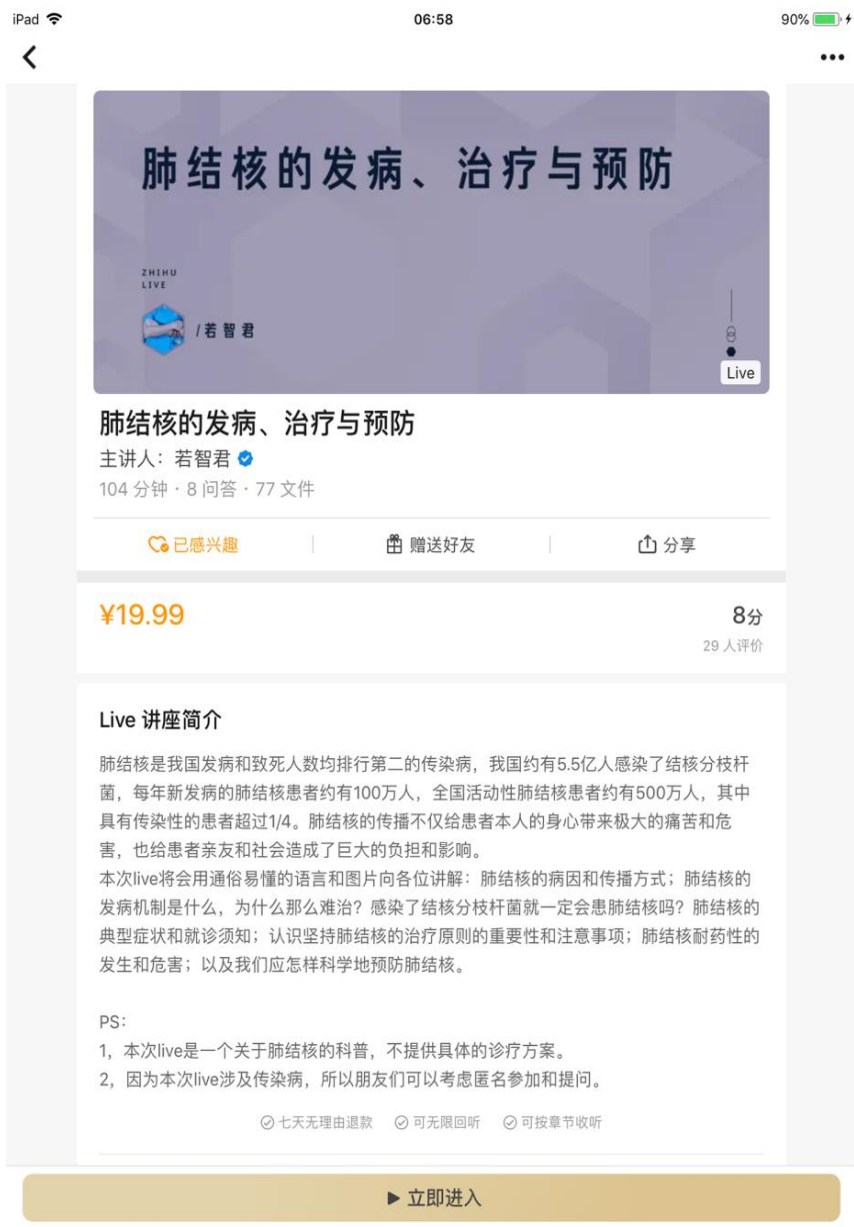

Figure 1: The ratings of the participants (in Chinese). As of June 2021, 29 participators had rated the live.

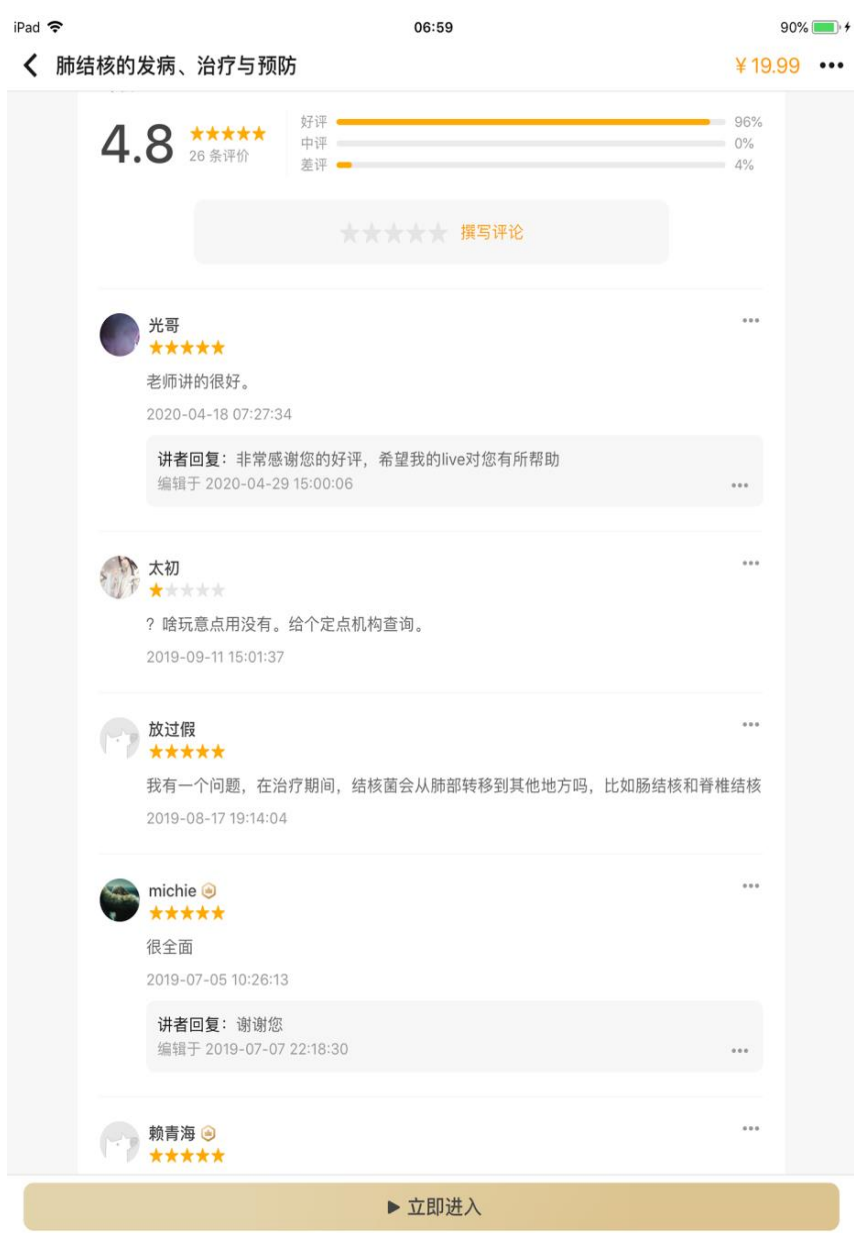

Figure 2: The comments of the evaluators (in Chinese).

Approximately twenty-six evaluators have commented on the Live lecture. Most reviewers showed great satisfaction with the content of the Live lecture and the speaker's performance.

\section{Discussion}

Since social media can be transmitted to a great number of people and therefore significantly spread health promotion messages, such media can be a powerful tool for health education. Through social media interventions, health behaviors among individuals can encourage positive health changes or help to prevent negative changes[6,12]. There are a variety of social media forms that can significantly spread health messages, including the internet and mobile phones. Some creative avenues can disseminate health information to specific target audiences[6,12]. Social media can change the behavior of whole populations through both direct and indirect pathways[13]. Individual recipients are directly affected by invoking cognitive or emotional responses[12]. Behavior can also change through indirect routes. First, the agenda of a discussion about a particular health issue within an individual's social network can be implemented by social media. Then, the frequency and/or depth of the discussion can be enhanced[12]. Since social media platforms reach a large number of audiences, behavioral changes within the public often become norms within an individual's social network. These norms influence a person's decisions, even when individuals are not exposed to social media[12]. The concurrent availability of and access to key services and products are very important for attracting individuals through media information and then encouraging them to take 
action[12]. Public relations or media advocacy can shape news and entertainment media campaigns that are used for public health issues[12]. Therefore, a new social media platform, such as Zhihu, might be a promising complementary strategy through which to publish conventional media campaigns for public health education.

The small amount of general knowledge about TB is a critical barrier to its control both domestically in China and worldwide [1,3]. Previous surveys have found poor TB awareness among the public in most regions of China $[3,14,15]$. Furthermore, previous studies have found that health education contributes greatly to improving TB knowledge and awareness, health-seeking behavior, and treatment adherence, thereby reducing the incidence of drug-resistant TB and TB infection[2,3,16]. However, only a few people had ever received a TB-related health education. A previous study found that inadequate TB-related health education might be the main cause of the low public awareness of TB in China[3].

Certain modes of health education are more useful for particular groups than others[17]. With conducting a needs assessment to understand the target population, we should develop more effective strategies with which to target specific population groups[17]. A previous study also suggested that the TB-related health education for populations at different socioeconomic levels, ages and education levels should be reassessed[3]. Furthermore, the developers of health education programs should rethink their current approach to this issue[3].

Whether worldwide or domestically in China, people in all age groups can be affected by TB; however, the highest burden is among adults. Furthermore, young and middle-aged individuals account for a considerable part of the adult TB incidence rate in China[1]. In terms of demographics, most Zhihu users are young, middle-aged, and highly educated[18,19]. These demographics suggests that the examined Zhihu Live lecture that focused on TB-related health education was mostly aimed towards these groups, regardless of whether they were young or middle-aged, especially highly educated TB patients or the people surrounding such patients. In addition, older patients account for most of the adult TB incidence rate in China[1]. For TB patients in China, relatives and friends are the main sources of their knowledge about TB[3]. We speculate that TB patients from all age groups and education levels will benefit from this Live lecture through the participants who surround them, such as a highly educated and young-adult son who would share health information that he gleaned from the lecture with his less-educated parents. This infers that not only all PTB patient groups and but also the public can benefit from this Live lecture. Furthermore, the public will benefit greatly from their improved understanding of TB gained from the lecture, regardless of the current prevention or treatment of TB.

The limitation of Zhihu Live is while since most TB patients worldwide live at the poverty level and are less educated, Zhihu Live lectures seem to mostly appeal to well-educated groups. However, to understand the needs and characteristics of the target populations, Zhihu Live on the Zhihu platform can be an effective tool for TB-related health education.
Furthermore, we speculate that in addition to TB, the Zhihu Live lecture series can also contribute greatly to the health education of other infectious diseases, including HIV/AIDS and COVID-19.

\section{Conclusion}

Zhihu Live can facilitate the effective learning and spread of knowledge regarding pulmonary tuberculosis for TB patients and those who surround them. The Zhihu Live lecture associated with PTB can be seen as a useful and innovative approach to public health education. In addition to TB, the prevention and treatment of other infectious diseases, including HIV/AIDS and COVID-19, could greatly benefit from this innovative health education method. The online community Q\&A platform including Zhihu and Quora can contribute greatly to public health education.

\section{Acknowledgments}

This study was supported by Guangdong Medical University Research Fund (grant numbers GDMUM 201818, GDMUM 2019022, GDMUM 2019036 and GDMUM 2020023); Zhanjiang City Non-funded Science and Technology Research Project (NO. 2020B01147 and 2021B01043); National Natural Science Foundation of China (grant number 81702285); Natural Science Foundation of Guangdong Province (grant number 2016A030313822); Guangdong Education Science "13th-five year plan" Research Project of 2020 (NO.2020GXJK412); Guangdong Province Educational Science Planning Project of 2021 (Higher Education Special Project) (NO. 2021GXJK468), The "14th Five-Year Plan" of the Guangdong Higher Education Association Higher Education Research Project of 2021(NO. 21GYB158). Guangdong Higher Education Reform Program (NO. 2018270)

The website of the Zhihu platform is as follows: https://www.zhihu.com/. The website of the Zhihu Live lecture used in our study is as follows: https://www.zhihu.com/lives/956901540947038208. You can also find this Zhihu Live lecture in the Zhihu app.

\section{Conflicts of Interest}

All authors have completed the ICMJE uniform disclosure form. The authors have no conflicts of interest to declare.

\section{Ethical Statement}

The authors are accountable for all aspects of the work in ensuring that questions related to the accuracy or integrity of any part of the work are appropriately investigated and resolved.

\section{References}

[1] “Organization WH. Global tuberculosis reports," 2020. [Onlline]. Available: https://apps.who.int/iris/bitstream/handle/10665/336069 /9789240013131-eng.pdf. 
[2] Gopu GS, Rao VB, Vadivet J, "Impact of health education on the knowledge of tuberculosis among sputum-positive pulmonary TB patients and their care-givers," The Nursing journal of India, 103(4), pp. 160-162, 2012.

[3] Chen W, Li Y, Yang H, et al, "Is tuberculosis health education reaching the public in China? A cross-sectional survey in Guizhou Province," BMJ open, 6(9), pp. e013534, 2016.

[4] Vernon A, Fielding K, Savic R, Dodd L, Nahid P, "The importance of adherence in tuberculosis treatment clinical trials and its relevance in explanatory and pragmatic trials," 16(12), pp. e1002884, 2019.

[5] Kigozi NG, Heunis JC, Engelbrecht MC, Janse van Rensburg AP, van Rensburg HCJD, "Tuberculosis knowledge, attitudes and practices of patients at primary health care facilities in a South African metropolitan: research towards improved health education," BMC Public Health, 17(1), pp. 795, 2017.

[6] Sight UF, "Module 2: Mass Media as an Educational Tool to Promote Health." Available: http://www.uniteforsight.org/health-education/module2.

[7] Alasmari A, Zhou L, "How multimorbid health information consumers interact in an online community Q\&A platform," International journal of medical informatics, 131, pp. 103958, 2019.

[8] Young I, Bhulabhai M, Papadopoulos A, "Safe Food Handling Advice Provided on Question-and-Answer Web Sites Is Inconsistent," Journal of nutrition education and behavior, 52(7), pp. 688-696, 2020.

[9] Charlie AM, Gao Y, Heller SL, "What Do Patients Want to Know? Questions and Concerns Regarding Mammography Expressed Through Social Media," Journal of the American College of Radiology: JACR, 15(10), pp. 1478-1486, 2018.

[10] Dudarenok AG, “Zhihu: China's Q\&A social platform that's ripe for brands." Available: https://econsultancy.com/zhihu-china-s-q-a-social-platf orm-that-s-ripe-for-brands/.

[11] Social D, "What is Zhihu? A Marketing Guide to the largest Q\&A platform in China." Available: https://www.dragonsocial.net/blog/zhihu-marketing-gui $\mathrm{de} /$.

[12] Wakefield MA, Loken B, Hornik RC, "Use of mass media campaigns to change health behaviour," The Lancet, 376(9748), pp. 1261-1271, 2010.

[13] Hornik R, Yanovitzky I, "Using Theory to Design Evaluations of Communication Campaigns: The Case of the National Youth Anti-Drug Media Campaign," Communication Theory, 13(2), 2003.

[14] Chen YLLQYXFHQCM, "The National awareness survey on key TB messages in 2010," Chinese Journal of Antituberculosis, 2013(1), pp. 60-64.

[15] Huang Y, Zhong JM, Chen SH, Chen B, Wang XM, "Investigation analysis on public awareness of tuberculosis knowledge in Zhejiang province, 2010," Chinese Journal of Preventive Medicine, 46(4), pp. 352-354, 2012.

[16] Alipanah N, Jarlsberg L, "Adherence interventions and outcomes of tuberculosis treatment: A systematic review and meta-analysis of trials and observational studies," Plos Medicine, 15(7), pp. e1002595, 2018.
[17] Saha A, Poddar E, Mankad M, "Effectiveness of different methods of health education: a comparative assessment in a scientific conference," BMC Public Health, 5, pp. 88, 2005.

[18] De Gennaro T, "10 Most Popular Social Media Sites in China (2020 Updated)."

[19] Achim A-L, "Why China's Q\&A Platform Zhihu is the Best Option for Beauty Brands," jingdaily.com, May 15, 2020. [Onlline]. Available: https://jingdaily.com/why-chinas-qa-platform-zhihu-is-t he-best-option-for-beauty-brands/. 